monopoles in experiment. The core of their analysis concerns the dynamical response of spin ice in the monopole picture - that is, the collective response of the magnetic moments to applied external magnetic fields. Crucially, this should depend on the peculiar properties of the excitations that are driven around the system by the applied field, and the literature already contains a range of detailed experimental studies of precisely such properties ${ }^{4,7-9}$.

The central result is the striking agreement between the spin autocorrelation time in the simulations and the timescale extracted from experiments on the a.c. susceptibility ${ }^{4}$ in one of the canonical spin-ice materials, $\mathrm{Dy}_{2} \mathrm{Ti}_{2} \mathrm{O}_{7}$ - agreement obtained with essentially only a single adjustable parameter, corresponding to the overall timescale of the dynamics. This finding provides compelling evidence of the role of monopoles as the fundamental and dominant excitations within spin ice, and it begins to paint a picture of their collective dynamics. Notably, it is the first quantitative signature of monopoles in a temperature-dependent dynamical quantity and contrasts with previous experimental justifications of the monopole's importance involving primarily equilibrium phase behaviour ${ }^{1}$. The expected magnetic
Coulomb interaction seems to be essential to explain the experimental results, because the predicted behaviour without such an interaction deviates from the experimentally observed timescale by orders of magnitude.

The growing body of evidence for monopoles in spin ice suggests the fascinating possibility that it may one day be possible to observe and even to manipulate individual monopoles in spin ice. More generally, however, Jaubert and Holdsworth's study raises the broader question of how the network of intertwined Dirac strings emanating from magnetic monopoles might manifest itself in other systems. One possible route of exploration might be through lithographically created (and thus highly versatile) microarrays forming twodimensional spin-ice analogues ${ }^{10}$ in which the individual magnetic dipoles can be probed; indeed, in these systems, the slow dynamics is also one of the most striking phenomena ${ }^{11}$. Another very recent proposal for the observation of monopoles in two dimensions uses not a magnet but the surface of a material containing bismuth - which realizes an exotic new phase known as the topological insulator - as a substrate for monopoles ${ }^{12}$.
For the time being, however, spin ice remains the only game in town for observing monopoles that are freely mobile in three dimensions, and Jaubert and Holdsworth's work provides an important step towards a detailed understanding of their dynamics.

Roderich Moessner ${ }^{1}$ and Peter Schiffer ${ }^{2}$ are at ${ }^{1}$ the Max-Planck-Institut für Physik komplexer Systeme, 01187 Dresden, Germany, and ${ }^{2}$ the Department of Physics and Materials Research Institute, Pennsylvania State University, 104 Davey Laboratory, University Park, Pennsylvania 16802, USA.

e-mail:moessner@pks.mpg.de; schiffer@phys.psu.edu

References

1. Castelnovo, C., Moessner, R. \& Sondhi, S. L. Nature 451, 42-45 (2008)

2. Bramwell, S. T. \& Gingras, M. J. P. Science 294, 1495-1501 (2001).

3. Jaubert, L. D. C. \& Holdsworth, P. C. W. Nature Phys. 5, 258-261 (2009)

4. Snyder, J. et al. Phys. Rev. B 69, 064414 (2004).

5. Ramirez, A. P., Hayashi, A., Cava, R. J., Siddharthan, R. \& Shastry, B. S. Nature 399, 333-335 (1999).

6. Siddharthan, R. et al. Phys. Rev. Lett. 83, 1854-1857 (1999).

7. Matsuhira, K., Hinatsu, Y. \& Sakakibara, T. J. Phys. Condens. Matter 13, L737-L746 (2001).

8. Ehlers, G. et al. Phys. Rev. B 73, 174429 (2006).

9. Clancy, J. P. et al. Phys. Rev. B 79, 014408 (2009)

10. Wang, R. F. et al. Nature 439, 303-306 (2006).

11. Möller, G. \& Moessner, R. Phys. Rev. Lett. 96, 237202 (2006).

12. Qi, X.-L., Li, R., Zang, J. \& Zhang, S.-C. Science 323, 1184-1187 (2009)

\title{
BIOPHYSICS
}

\section{Nothing without water}

Proteins can perform a remarkable variety of functions, including the catalysis of biochemical reactions, cell signalling and the transport of molecules. However, Hans Frauenfelder and colleagues suggest that, on their own, these 'picomachines' are essentially stalled - what brings them to life is the surrounding water, which acts as a kind of puppeteer controlling the protein dynamics (Proc. Natl Acad. Sci. USA doi: 10.1073/ pnas.0900336106; 2009).

The interaction between a protein and its environment happens on two levels: on the one hand there is the bulk solvent, on the other the hydration shell (made up of the water molecules that interact directly with the protein). In their 'unified model of protein dynamics', Frauenfelder et al. propose that fluctuations in the hydration shell dictate internal protein motions, whereas fluctuations in the bulk environment control large-scale motions of the protein, such as shape changes.
In water, as in other glass-forming liquids, there are two qualitatively different types of equilibrium fluctuation, which originate from the bulk solvent and the hydration shell, respectively. Furthermore, the energy landscape of proteins - which defines the conformations a protein can assume - is organized hierarchically. To take account of both of these factors, Frauenfelder et al. associate the two types of fluctuation with random walks in different tiers in the highly structured energy landscape. As the fluctuations in the bulk water are controlled mainly through its viscosity (which is believed to be quite constant in cells), the range of possible large-scale motions that these fluctuations can control seems relatively narrow. The hydration shell, in contrast, is highly structured, implying that many possible internal motions can be induced.

Frauenfelder et al. have compared their predictions with experimental data on the dynamics of the biomolecule myoglobin (pictured), and have found them to be consistent. Their model correctly predicts the motion of carbon monoxide through this protein and the relaxation of the protein structure after the carbon monoxide has been released. Nevertheless, they advise caution because there are other motions, not only the dynamical processes considered in their study, that are important in protein function.

ANDREAS TRABESINGER 